\title{
QUANTITATIVE MEASUREMENTS OF LOCAL IRREGULARITIES IN WOVEN FABRICS BY OBSERVING OPTICAL DIFFRACTION PATTERNS
}

\author{
By Ryuichi Akiyama, Toshihiro Iguro, Sei Uchiyama \\ Hideo Noshi and Juzo Hosokawa \\ (Faculty of Textile Fibers, Kyoto Institute of Technology, \\ Matsugasaki, Sakyo-ku, Kyoto 606, Japan)
}

\begin{abstract}
The local irregularities of a fabric were obtained by measuring peak intensities of the diffraction pattern. Numerical analyses have predicted that these peak intensities are given uniquely as a function of the statistical deviations of the irregularities of a yarn coarseness and a yarn arrangement, $\sigma_{\alpha}$ and $\sigma_{\beta}$, as a parameter of the ratio $(R)$ between the yarn coarseness and the yarn spacing. Experimentally, the combinations of the above two deviations, $R^{2} \sigma_{a}^{2} / 4+\sigma_{\beta}^{2}$, were determined over a range of 4 to $10 \%$ within an accuracy of $\pm 1 \%$ by using commercial acetate and cotton fabrics. The experimental results of total deviations of these two irregularities were in good agreement with those obtained from manual measurements. Correlation between the combination of two deviations and visual sensory property was also made.
\end{abstract}

\section{Introduction}

A woven fabric has the local irregularities of a yarn coarseness and the spacing between neighboring two yarns, i.e., yarn arrangement. These two irregularities are distributed at different levels in a fabric. Each of them has been evaluated by obtaining the mean value or standard deviation directly from manual measurements. There are two methods with respect to the measurements on the irregularity of the yarn coarseness: Uster evenness tester method and seriplane method. But, in the case of the irregularity of the yarn arrangement, the ordinary one employs tedious manual measurements as a method for measuring the cloth count, although there are a few studies on the pattern recognition of the fabric structure based on the computer processing. ${ }^{1-4)}$ The product test of commercial woven fabrics has been also, in practice, done by using the visual sensory method.

Optical diffraction method can remedy these drawbacks. The diffraction pattern of the fabric depends on the structural properties. One of our authors has already discussed the details on the diffraction pattern of the regular fabric structure in a previous paper. ${ }^{5)}$ And he has also described the possibility of the detection of the remarkable periodic irregularities of the yarn arrangement, for example, a missing or insertion defect. ${ }^{6}$ )

As a last step, a small amount of the irregularities of the fabric structure will be discussed. To make these local irregularities evaluate numerically, there remain at least two questions to be answered: (1) To what extent can we explain the relation between the two irregularities of a yarn coarseness and a yarn arrangement, and the diffraction pattern theoretically and experimentally? (2) How can the experimental evaluation be correlated to the visual sensory test? The aim of this paper is to answer some aspects of these questions by studying the optical diffraction pattern of the woven fabric experimentally.

\section{Relation Between Principal Maximum and Irregularity}

We will consider one dimensional array that contains a large number of similarly oriented yarns 


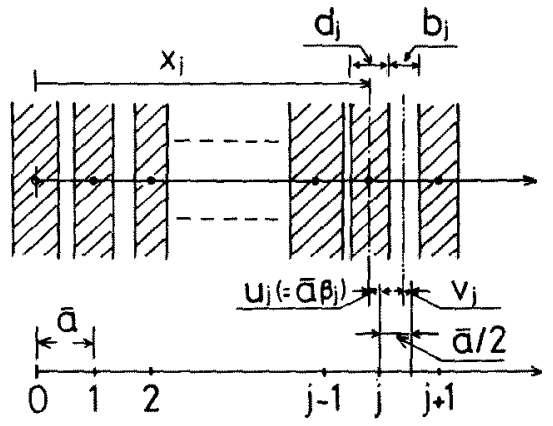

Fig. 1. Schematic illustration of a fabric model having the irregularities of a yarn coarseness and a yarn arrangement, $d_{j}$ and $u_{j}$, respectively. $b_{f}$ and $v$, indicate the irregularities of yarn interstices and their arrangement.

as shown in Fig. 1. Let the coordinates at the center of the yarn refered to the fixed axis be $x_{0}(=0), x_{1}, \ldots, x_{j}, \ldots$ Let the mean value of the periodic interval between neighboring two yarns, and the irregularity of the center of the yarn relative to the normal coordinate be $\bar{a}$ and $\bar{a} \beta_{j}$, respectively. The relation is given as follows

$$
x_{j}=j \bar{a}+\bar{a} \beta_{j} .
$$

And let $\bar{d}$ and $\bar{d} \alpha_{j}$ be the mean coarseness of the yarns and their irregularity, respectively. The $j$-th yarn coarseness is given by

$$
d_{j}=\bar{d}\left(1+\alpha_{j}\right) .
$$

Here $\alpha_{y}$ and $\beta_{j}$ are given as the averages of a set of the random values over a range of from -0.5 to 0.5 . Central limiting theorem indicates that the statistical properties of these averages obey Gaussian distribution. And their variances decreases monotonically, that is, the shape of the Gaussian distribution becomes sharp, as the total number of random variables increases.

By using previous notations ${ }^{5}$, the one-dimensional intensity distribution diffracted from the irregular structure of the fabric model is given by

$$
\begin{aligned}
& I(p)=\operatorname{const}\left|\sum_{j=0}^{N-1} d_{j} \cdot G_{j}(p) \cdot \exp \left(-i k x_{j} p / F\right)\right|^{2}, \\
& G_{j}(p)=\sin \left(k d_{j} p / 2 F\right) /\left(k d_{j} p / 2 F\right)
\end{aligned}
$$

Here $k(=2 \pi / \lambda)$ is wave number, $\lambda$ wavelength, $F$ the distance between the object and the diffracted planes. The first term, $G_{f}(p)$, represents the diffracted wave of a single yarn, and $p$ denotes the coordinate axis in the diffracted plane. The principal maxima are separated by an amount of $m(\lambda F / \bar{a})$ in the diffraction pattern.

In order to obtain the dependence of the principal maximum on the irregularity of the yarn coarseness and the yarn arrangement, we calculated the intensity ditribution of the complimentary screen of the actual fabric. Since the incident light transmits the geometrical interstices between the yarns in our experiments, it is natural that the intensity distribution of eq. (3) should be expressed by using the yarn interstices $b_{f}$ and their periodic spacings $u_{j}$. From the Babinet's principle ${ }^{7)}$ we can obtain the same distribution of light diffracted by the complimentary screens i.e. by screens which are such that openings in one correspond exactly to opaque portions of the others and vice versa except in the intermediate neighborhood of the image of the source.

The quite different intensity distributions are obtained, depending on whether the irregularity is large or not. These irregularities have an effect on the broadness of the principal maxima in the diffracted plane. It is possible to evaluate the broadness numerically by the following two methods. One is to measure the half width of the principal maximum and the other is to measure the peak value of the principal maximum. The disadvantage of the half width measurements is modest sensitivity experimentally. We will describe the relation between the peak values of the principal maxima and the irregularities.

In general, the peak values of the principal maxima decrease monotonically, with increasing the irregularities of the yarn coarseness and the yarn arrangement. By using eqs. (1) to (4), we calculated the peak intensity integrated over a small range of $10^{-3}$ in comparison to the peak interval. By substituting $m(\lambda F / \bar{a})$ in place of $p$ into eq. (3), the peak values of principal maxima are given by

$$
\begin{aligned}
I(m)=I(0) \cdot \mid \sum_{j=0}^{N-1} \frac{\sin \left(m \pi R\left(1+\alpha_{j}\right)\right)}{m \pi R\left(1+\alpha_{j}\right)} \\
\left.\cdot \exp \left(-i 2 \pi \beta_{j} m\right)\right|^{2} \\
=I(0) \cdot\{\sin (m \pi R) / m \pi R\}^{2} \cdot I_{0}(m) .
\end{aligned}
$$


Here the integer $m$ denotes the order of the diffracted intensity and $R$ is the ratio of $\bar{d}$ to $\bar{a}$, i.e., the tatio between the averages of the yarn coarseness and the periodic spacing of the yarn arrangement. The first term in eq. (6), $I(0)$, is the central intensity and the last term $I_{0}(m)$ depends on the irregularity. It is normalized so as to become unity when the fabric has no irregularity.

Fig. 2 shows the normalized intensity $I_{0}(1)$ as a function of the two standard deviations of the yarn coarseness and the yarn arrangement $\sigma_{\alpha}$ and $\sigma_{\beta}$, respectively. One dot was calculated by using the following parameters, $N=200$ and $R=0.75$, when $m=1$. The calculated result shows that the best fit lines for the dots can be expressed as a simple function, although the intensity is influenced by the superposition of these two irregularities. The equation (5) indicates that the peak intensities of the principal maxima depend not on how yarns are arranged, but on what the two deviations of the irregularities are. The peak intensity is determined uniquely when the fabric has the same deviation.

The last term in eq. (6), $I_{0}(m)$, is rewritten as follows to a first approximation over a range of small deviation.

$$
\begin{aligned}
I_{1}(m) & =\left(1-2(m \pi)^{2} \cdot \sigma^{2}\right)^{2}, \\
\sigma^{2} & =R^{2} \sigma_{\alpha}^{2} / 4+\sigma_{\beta}^{2}
\end{aligned}
$$

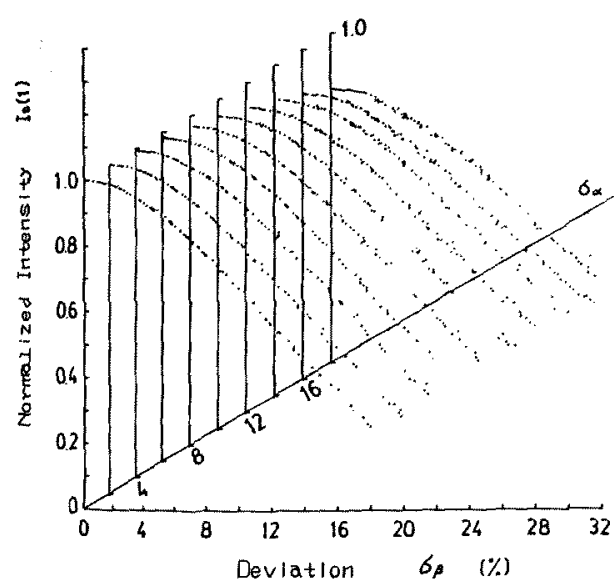

Fig. 2. Peak intensities plotted as a function of the deviations of the irregular fiber coarseness $\left(\sigma_{\alpha}\right)$ and the irregular yarn arrangement $\left(\sigma_{\beta}\right)$ over a range from 0 to $20 \%$ as a parameter of $R=0.75$ at $m=1$.
The solid lines in Fig. 3 were calculated from eq. (7) when $R=0.25$. One dot denotes the peak intensity at the $m$-th diffracted order calculated from the last term in eq. (6). The relation given by eq. (7) holds in the range of $\sigma<12 / m \%$. The local irregularity of the woven fabric can be evaluated numerically by the total deviations $\sigma$ in the case of diffraction method.

Fig. 4 shows the intensity contours as a function of $\sigma_{\alpha}$ and $\sigma_{\beta}$ for a fixed ratio $R=0.75$. It is only the total deviation that we can obtain from

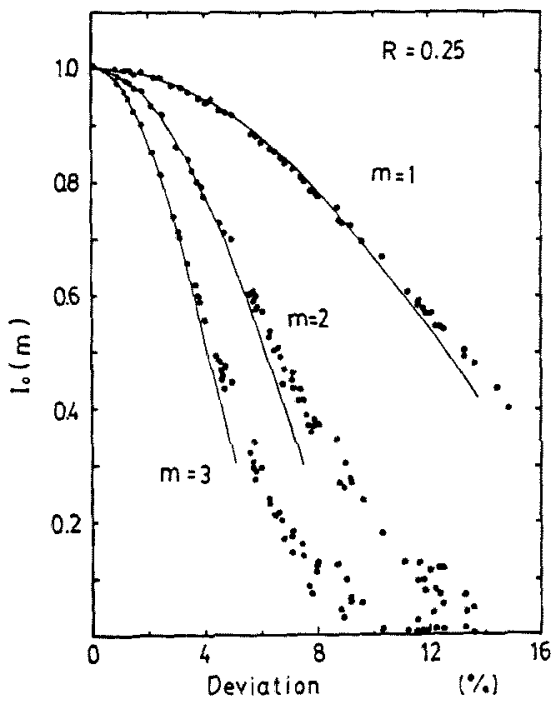

Fig. 3. Relation between the diffracted intensity calculated from eq. (5) and the total deviation by eq. (8). Solid lines denote the first-order approximation curve of eq. (5).

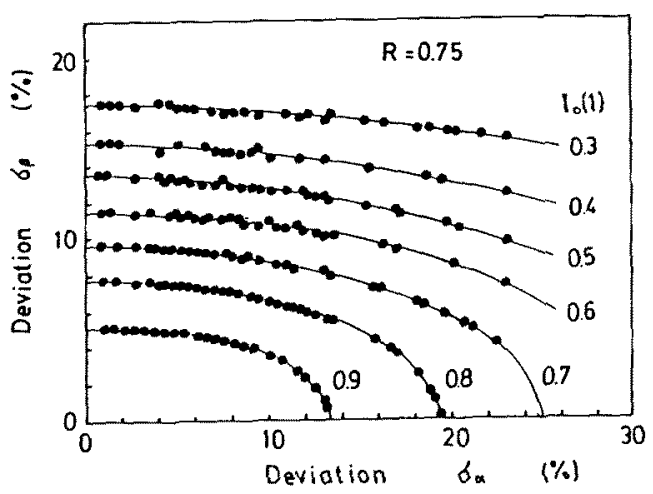

Fig. 4. Intensity contours for the peak intensities, $I_{0}(1)$ plotted as a function of $\sigma_{\alpha}$ and $\sigma_{\beta}$. 
the measurements of the diffracted intensity. Each of the two deviations is not determined separately. The total deviation is regarded approximately as the deviation of the periodic irregularity, since the contribution of the yarn coarseness is $R^{2} / 4=1 / 7$ as in eq. (8) even when the fabric having $R=3 / 4$. The intensity of $I_{1}(m)$ depends strongly on the irregular periodic spacings.

\section{Experimental Procedures}

The samples used in our experiments were commercial plain woven fabrics. They have different irregularity patterns of a yarn coarseness and a yarn arrangement. Optical measuring system was similar to that used previously to study the detection of the defects in a fabric. $\left.{ }^{6}\right)$ The intensity measurements were made by using a CCD image sensor and a photomultiplier tube. The output data from the CCD detector were stored into the memory of a microcomputer (Apple II and/or PC8001MK-II) after being passed through an interface consisting of a timing circuit and a digitizer. The samples were illuminated by a plane wave from 20 to $50 \mathrm{~mm}$ in diameter. There are at least several hundreds of the yarn interstices of the fabric in the area.

\section{Results and Discussion}

In the previous section, the complimentary screen of the fabric has been used to obtain the intensity distribution reflected directly on the irregularities of the yarn coarseness and the yarn arrangement. In order to compare the experimental results and the calculated ones of the actual fabric, the intensity distribution of the actual fabric has been calculated in what follows. The peak values of the principal maxima are given by

$$
I^{\prime}(m)=I^{\prime}(0) \cdot\left\{\sin \left(m \pi R^{\prime}\right) /\left(m \pi R^{\prime}\right)\right\}^{2} \cdot I_{1}(m),
$$

An average of the ratio between the yarn interstices and yarn spacing $R^{\prime}(=1-R)$ is used in the second term of eq. $\left(6^{\prime}\right)$. Note that the intensity of $I_{1}(m)$ can be expressed by using the ratio $R$, not $R^{\prime}$. Naturally, the intensity $I_{1}(m)$ is also
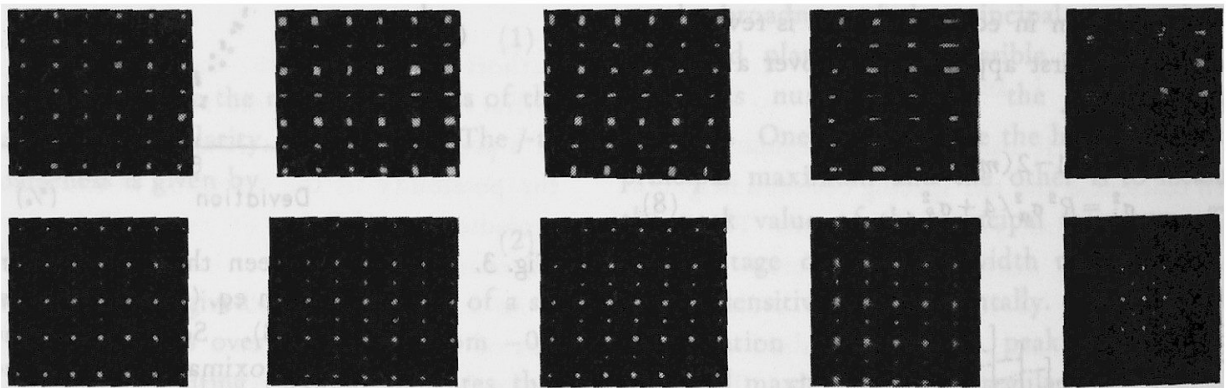

(c)

(d)

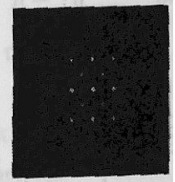

(a)
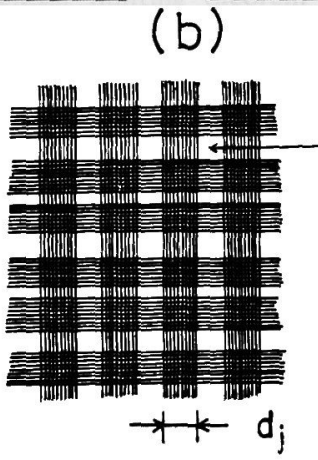

interstices
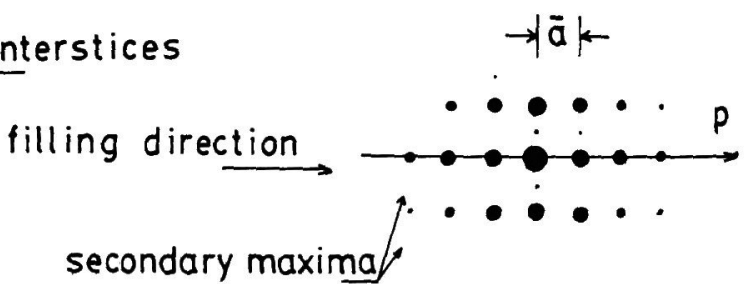

(f)

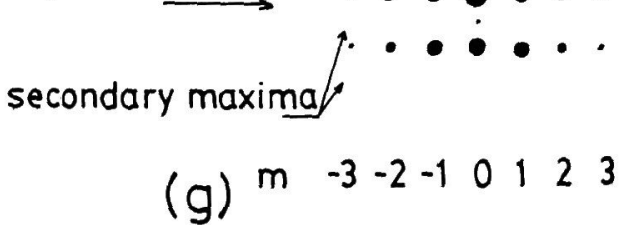

Fig. 5. Photos of the transmitted images of fabrics on the upper side and their diffraction patterns on the lower side. Samples are four types of acetates (a to d) and cotton (e). (f) and (g) are schematic illustrations of photo images and diffracted patterns, respectively. 
written by the irregularities of the fabric interstices and their arrangement. Since these irregularities results from the yarn coarseness and the yarn arrangement, the corresponding intensities are expected to be equal.

Figs. 5 show the transmitted images of the acetate fabrics (a to d) and a cotton fabric (e) under microscopic field on the upper side. Photos $a$ to $d$ are arranged in the order of the deviation of the periodic irregularity. Several different irregularities ( $b$ to $d$ ) were obtained by applying the small external disturbance to the fabric of a. It is rather difficult to find out the differences between the photos $a$ and $b$. Visual sensory test requires to detect the irregularities to be greater than $10 \%$. The increment of the irregularity results in the broadening and/or the reduction of the diffracted intensity at the side of higher order as shown in Figs. 5, especially photo d. The fuzziness is not observed in the acetate fabrics.

To confirm the results described in the previous section experimentally, the intensity measurements were done at the $m$-th order of the diffraction pattern. The peak values of the principal maxima were obtained along the filling-direction as indicated by an arrow in Figs. 5. Considerable care was taken to the symmetry of the intensity distribution of the diffraction pattern and the significance of the peak value at the zero order.

The total deviations of the woven fabrics are determined from the last term of eq. $\left(6^{\prime}\right)$. There are two unknown parameters in eq. (6). One is the ratio of $R^{\prime}$ and the other is the total deviation itself. The parameter of $R^{\prime}$ can be determined by observing directly the zero intensity of the second term of eq. $\left(6^{\prime}\right)$ in the diffraction pattern. Since a number of $m$ peak intensities can be measured, two or three relationships as in eq. $\left(6^{\prime}\right)$ are at least obtained. Therefore it is not difficult to determine these two unknown parameters. They were obtained experimentally from the least-square fitting procedure.

Fig. 6 shows the least-square fitting procedure for the last term in eq. (6) graphically. By substituting the best fit value of the ratio $R^{\prime}$ into eq. $\left(6^{\prime}\right)$, one can calculate the last term in eq. $\left(6^{\prime}\right)$. The total deviation was given by the point of intersection of the dotted line and the last term in eq. $\left(6^{\prime}\right)$. Open circles denote the points of the

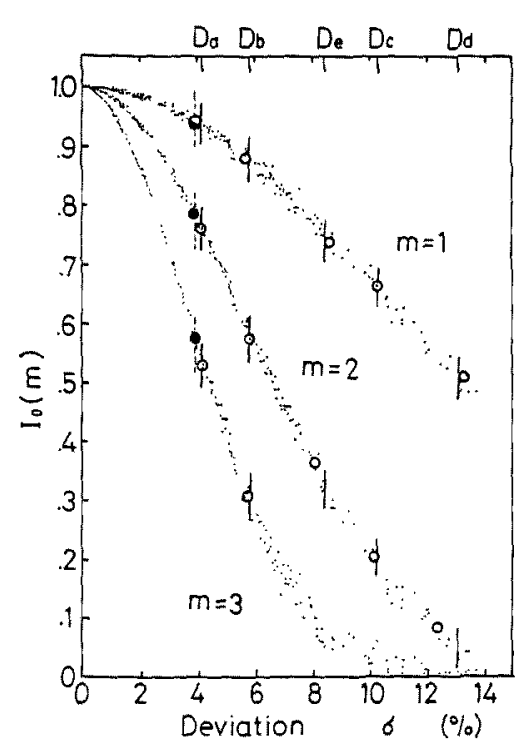

Fig. 6. Relation between observed intensities and total deviations $\sigma$ when $m=1,2$ and 3 . Open circles denote the deviations determined from the experimental intensities. Each deviation of the woven fabrics, a to e, are determined from the least-square fitting procedures for the combination of three intensities. Closed circles indicate the intensities obtained from manual measurements. For details, see the section of visual sensory property.

intersection when $m=1,2$ and 3 . The undersubscripts of the deviations (D), a to e, stand for the fabrics a to $\mathrm{e}$ in Figs. 5. There are slight differences among the deviations obtained from the peak values of $m=1,2$ and 3. These uncertainties are mainly due to the inhomogeneous yarn interstices, such as the yarn orientation and the fuzziness of the fibers. The discrepancy as shown in the sample $\mathrm{e}$ is responsible for the influence of the fuzz effect (photoe). The measurements of the fuzz effect will be discussed elsewhere in the future. The sub-peak intensities observed parallel to the filling direction as in Figs. 5 will be also reported.

Table 1 shows the experimental values of the averages of the yarn coarseness and the periodic spacing, $\bar{d}$ and $\bar{a}$, and their standard deviations with respect to sample a. They were in good agreement with those of the manual measurements (obs.). It can be concluded that the standard 
Table 1. Comparison between results obtained from optical diffraction measurements and manual measurements. Standard deviations $a$, and averages of yarn spacing $\bar{a}$ and ratios of $\bar{d} / \bar{a}(=R)$ are listed.

\begin{tabular}{|c|c|c|c|c|c|r|}
\hline Sample & \multicolumn{2}{|c|}{$\bar{a} \mathrm{~mm}$} & \multicolumn{2}{|c|}{$R$} & \multicolumn{2}{c|}{$\sigma$} \\
\hline No. & exp. & obs. & exp. & obs. & exp. & obs. \\
\hline a & 0.32 & 0.32 & 0.18 & 0.19 & 4 & 5 \\
b & 0.32 & 0.32 & 0.19 & 0.21 & 6 & 6 \\
c & 0.32 & 0.32 & 0.18 & 0.20 & 10 & 9 \\
d & 0.32 & 0.32 & 0.19 & 0.20 & 13 & 14 \\
e & 0.38 & 0.38 & 0.36 & 0.38 & 8.5 & 7 \\
\hline
\end{tabular}

deviation of the local irregularities of the woven fabric can be determined over a range of 4 to $10 \%$ to an accuracy of the order of $\pm 1 \%$. Optical diffraction method is good to detect the small amount of the irregularities in the two dimensional lattice structure.

\section{Visual Sensory Property}

The rays of the incident light on the woven fabric with a high cloth count, give dark stripe lines frequently in the transmitted pattern. These defects are observed especially along the warp direction even when the irregularities of the yarn coarseness and the yarn arrangement are small. This is one of the defects of the fabric structure, refered to as yarn streakiness.

The streakiness defect depends on the yarn interstices of the fabric structure. Let the $j$-th geometric interstices and its irregularity be $b_{j}$ and $v_{j}$ as in Fig. 1. By using eq. (1) and (2), the following relations are obtained.

$$
\begin{aligned}
& b_{j}=\bar{b}+v_{j}=\bar{a} R^{\prime}\left(1+r_{j}\right), \\
& \sigma_{\gamma}^{2}=\left(R^{2} \sigma_{\alpha}^{2} / 4+\sigma_{\beta}^{2}\right) / R^{\prime 2}
\end{aligned}
$$

Here $\sigma_{r}^{2}$ is the variance of the yarn interstices. The irregularity of the geometrical interstices increases relatively, with increasing the ratio $R$. The calculated results indicate that it is difficult to remove such defects in the woven fabric of a high cloth count even though the regular yarns and the good weaving machine were used. These defects are not observed in the small count of cloth, that is, when the ratio of $R$ is small, for example, gauze weaves. To a considerably high extent, the detection of the defect is influenced by the relative sensitivity of the human visual property. The product test of the commercial woven fabrics has been done by the visual sense of the skillful experts. In practice, there are some problems to be solved; (1) What parameters can be chosen in the sensory test? and (2) How can we make an numerical index of the sensory test?

The variance of $\sigma_{r}^{2}$ in eq. (10) depends on the total deviation, although the propotinality constants are not equal. These irregularities are similar to those of the diffraction method. The normalized intensities $I_{1}(m)$ can be calculated by using the total variances obtained from the variance of geometric interstices, $\sigma_{r}^{2}$ The calculated results are plotted as closed circles in Fig. 6. The agreement between the calculated results and experimental intensities are good. Therefore, it is advantageous to estimate the visual properties by measuring the peak intensities due to a small amount of the irregularities, when the experts watch the geometrical interstices of the fabric in the product test. Diffraction method can be utilized for the visual sensory test.

\section{Acknowledgments}

This work was supported in part by Takesi Sakurada Award in 1984.

It is pleasure to thank to Mr. Takami who did the computer programming with enthusiasm.

\section{References}

1) K. Furuta and E. Kuze; Sen-i Gakkaishi, 29, T-224 (1973).

2) L. Tsu-Ping, M. Sezako and E. Kuze; Sen-i Gakkaishi, 38, T-438 (1982).

3) L. Tsu-Ping, M. Sezako and E. Kuze; Sen-i Gakkaishi, 39, T-252 (1983).

4) K. Ohta, K. Sakaue and H. Tamura; Sen-i Gakkaishi, 36, 459 (1983).

5) R. Akiyama and E. Kuze; Sen-i Gakkaishi, 33, T-439 (1977).

6) R. Akiyama and E. Kuze; Sen-i Gakkaishi, 35, T-202 (1979).

7) M. Born and E. Wolf; Principles of Optics, 5ed., (Pergamon Press 1975), p381. 
光回折パターンの観測による織物構造のゆらぎの定量化

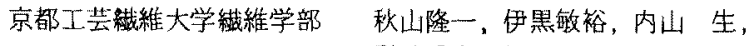

整斗秀夫，細川重三

織教らは系の太さ之系の配列周期の不均一性に依存す る。光の回折强度を剆定し，この織志らを定量化した。 上の 2 つの統計的な不均一性の標準偏差をそれぞれ， $\sigma_{\alpha}$ $c \sigma_{\beta}$ とし，系の平均周期に対する糸の平均太さの比を $R$ とすると，回折強度のピーク值は $R^{2} \sigma_{\alpha}^{2}+\sigma_{\beta}^{2}\left(=\sigma^{2}\right)$ 增 減化より単調变化することが，数值解析からあとから
れた。寒騃では、市貶の布を用いて回折強度を測定し， とのるを 4ー10\%の籍囲で，精度士 $1 \%$ で決めた。織物 分解の䊅果と比較すると，良く一致していた。また人が 織むらを評価する際の視覚特性上回折から得られた結果 との相関についても議論した。 